\title{
marges Marges
}

revue d'art contemporain Revue d'art contemporain

$11 \mid 2010$

Valeur(s) de l'art contemporain

\section{Hans Ulrich Obrist, A Brief History of Curating}

Zürich/Dijon, JRP|Ringier/Les Presses du réel, 2008

\section{Jérôme Glicenstein}

\section{(2) OpenEdition}

\section{Journals}

Édition électronique

URL : http://journals.openedition.org/marges/483

DOI : $10.4000 /$ marges.483

ISSN : 2416-8742

Éditeur

Presses universitaires de Vincennes

\section{Édition imprimée}

Date de publication : 15 octobre 2010

Pagination : 152-153

ISBN : 978-284292-262-7

ISSN : $1767-7114$

Référence électronique

Jérôme Glicenstein, « Hans Ulrich Obrist, A Brief History of Curating », Marges [En ligne], 11 | 2010, mis en ligne le 15 octobre 2010, consulté le 22 septembre 2020. URL : http://journals.openedition.org/ marges/483 ; DOI : https://doi.org/10.4000/marges.483 


\section{Hans Ulrich Obrist}

\section{A Brief History of Curating}

\section{Zürich/Dijon, JRPIRingier/Les Presses du réel, 2008, 239 p.}

Hans Ulrich Obrist est connu à la fois comme commissaire d'exposition - ce qu'il a longtemps été au sein du Musée d'art moderne de la Ville de Paris - et comme auteur d'un grand nombre d'entretiens pour des revues d'art contemporain. Dans le cadre de cette deuxième activité, Obrist a publié plusieurs dizaines d'ouvrages de "conversations" avec toutes sortes de personnalités (de Robert Crumb à Philippe Parreno, en passant par Gilbert et George). En dépit de cet engagement important et multiple, on a souvent l'impression qu'Obrist lui-même se place en retrait sans véritablement livrer son point de vue. C'est en cela que la publication de $A$ Brief History of Curating prend tout son intérêt, en ce qu'elle réconcilie ses deux principales activités tout en lui offrant, peut-être pour la première fois, l'occasion d'exprimer des positions un tant soit peu personnelles. À première vue, le livre ressemble à une suite d'hommages rendus à des commissaires d'exposition âgés ou déjà disparus. Onze d'entre eux ont été choisis, afin d'amorcer une histoire du commissariat d'exposition qui resterait à écrire. La liste de ces personnes pose tout de même quelques questions. D'une part, leur importance pour une histoire de l'exposition semble assez inégale. On s'interroge un peu sur les choix de Franz Meyer, Anne d'Harnoncourt ou Walter Zanini. D'autre part, elles ont fait preuve de démarches de nature très différente. Qu'y a-t-il de commun entre la pratique du conservateur Werner Hofmann et celle de l'activiste Lucy Lippard ? Entre le défenseur du rôle social du 
musée Pontus Hulten et Harald Szeemann, le chantre de la singularité irréductible des artistes ? À un moment, Obrist explique que les deux seules personnes ayant refusé d'être interviewées avaient été Jean Clay et Kynaston McShine (p. 215). Pourtant, ainsi que le note Daniel Birnbaum, dans sa postface, on pourrait aussi bien s'interroger sur l'absence de Kasper König ou Suzanne Pagé - les deux personnalités qui ont le plus soutenu Obrist à ses débuts. Voire sur l'absence d'autres acteurs particulièrement importants de l'histoire du commissariat d'exposition des quarante dernières années : William Rubin, Jean Clair, Norman Rosenthal, Rudi Fuchs, Jan Hoet, Achille Bonito Oliva, Germano Celant, Jean-Hubert Martin, Okwui Enwezor, Catherine David (entre autres).

La raison de ces absences apparaît peut-être en filigrane dans les questions formulées par Obrist. Ce qui l'intéresse, semble-t-il, c'est le flux de l'événement : le fait de déclencher des processus - créer des situations pour que des événements se produisent - ; le musée considéré comme un laboratoire. «Laboratorium » était d'ailleurs le titre d'une de ses expositions et les idées de processus ou d'événement formaient la substance de deux de ses projets les plus personnels : " do it » - un livre d'instructions pour réaliser des œuvres d'art ayant donné lieu à une série d'expositions - et "Utopia Station» - une suite d'expositions rassemblant de manière désordonnée des propositions " utopiques ». De fait, les intérêts personnels d'Obrist font écho à un certain nombre d'expositions organisées par les personnes auxquelles il s'adresse. C'est le cas de l'exposition fourre-tout "Thirty-Six Hours ", organisée par Walter Hopps à Washington (proposition qui n'est pas sans rappeler «Utopia Station »), de « Quand les attitudes deviennent forme » de Harald Szeemann, de

la biennale " démocratique " de Sao Paulo de Walter Zanini ou des différents projets de Seth Siegelaub. C'est un peu comme si Obrist cherchait à faire valider ses propres positions par ses interlocuteurs.

Le même phénomène se retrouve, lorsqu'il pose la question des modèles suivis par les uns et les autres; mettant en avant une influence qu'il croit grande chez tous, celle d'Alexander Dorner, le directeur du Landesmuseum de Hanovre dans les années 1930. Visiblement The Way Beyond Art a joué un rôle important dans sa formation - ou tout au moins la compréhension qu'il a de Dorner en tant que promoteur de l'idée de musée comme " centrale électrique " et laboratoire d'idées nouvelles. Mais la plupart des interlocuteurs d'Obrist refusent de le suivre sur ce terrain. Au contraire, sept d'entre eux (sur onze) mettent plutôt en avant l'influence de Willem Sandberg (du Stedelijk Museum d'Amsterdam). L'ironie de la chose c'est que la position de Sandberg - lequel a été jusqu'à défendre l'idée du conservateur de musée en tant qu'auteur - semble peu compatible avec l'idéal d'effacement du commissaire généralement promu par Obrist.

L'ouvrage d'Obrist tient-il ses promesses d'être une " brève histoire du commissariat d'exposition »? De fait, il est fait référence à un grand nombre d'expositions. Mais la forme des entretiens empêche d'entrer vraiment dans les détails. Ce qui fait qu'on a surtout une suite d'anecdotes et de remarques assez générales et superficielles : une sorte de doxa ou de digest de l'histoire de l'exposition qui permet surtout d'avoir assez rapidement des idées toutes faites sur le sujet. On l'aura compris, on reste un peu sur sa faim.

\section{Jérôme Glicenstein}

